\title{
Philosophiques
}

\section{Literal Meaning de François Recanati}

\section{Steven Davis}

Volume 33, numéro 1, printemps 2006

Philosophie et psychopathologie

URI : https://id.erudit.org/iderudit/012958ar

DOI : https://doi.org/10.7202/012958ar

Aller au sommaire du numéro

Éditeur(s)

Société de philosophie du Québec

ISSN

0316-2923 (imprimé)

1492-1391 (numérique)

Découvrir la revue

Citer ce document

Davis, S. (2006). Literal Meaning de François Recanati. Philosophiques, 33(1), 263-274. https://doi.org/10.7202/012958ar d'utilisation que vous pouvez consulter en ligne.

https://apropos.erudit.org/fr/usagers/politique-dutilisation/ 


\title{
Literal Meaning de François Recanati
}

\author{
STEVEN DAVIS \\ Université de Carleton \\ davis@connect.carleton.ca
}

Une des questions centrales du livre de François Recanati, Literal Meaning, concerne la façon de déterminer ce qui est dit lorsqu'on utilise ${ }^{1}$ une phrase dans un contexte donné ${ }^{2}$. Recanati affirme que ce qui est dit est déterminé par le contexte dans lequel la phrase est utilisée, même si celle-ci ne contient pas d'éléments indexicaux apparents ${ }^{3}$. Lorsque les phrases sont utilisées de façon assertive, ce qu'elles disent - leur signification, que Recanati assimile à leurs conditions de vérité - est déterminé par le contexte ${ }^{4}$. Il s'ensuit qu'il n'y a aucun usage d'une phrase ${ }^{5}$ assertive ${ }^{6}$ pour lequel les conditions de vérité seraient simplement données par la signification lexicale des parties et la forme logique de la phrase. Recanati affirme que différents processus pragmatiques en jeu dans l'interprétation déterminent ce qui est dit - c'est-à-dire, fournissent les conditions de vérité des phrases utilisées lors d'occasions particulières. Je soutiendrai

1. Pour Recanati, l'« utilisation » d'une phrase signifie l'accomplissement d'un acte de langage par énonciation de la phrase (p. 159). Dans ce qui suit, lorsque je parle de l'utilisation de phrases, je fais donc référence aux actes de langage.

2. La théorie de Recanati a beaucoup de points en commun avec la théorie de la pertinence de Sperber et Wilson (1986), et de Carston (2002), bien qu'elle diffère de celle-ci sur plusieurs points importants.

3. Dans la première partie de son livre, Recanati affirme que « suivant la conception à laquelle nous aboutissons, l'interprétation véri-conditionnelle est dans une large mesure pragmatique » (p. 21). Recanati semble donc être un contextualiste. Mais dans la dernière partie, il adopte ce qui semble être une proposition beaucoup plus modeste, le contextualisme méthodologique, à savoir que « toute expression peut, à l'analyse, s'avérer dotée d'une sémantique sensible au contexte, c'est-à-dire être telle que sa contribution aux conditions de vérité varie selon les contextes. Que cela soit le cas ou non pour une instance particulière relève de l'analyse empirique et non pas de stipulation a priori" (p. 160). En adoptant le contextualisme méthodologique, Recanati ne répudie pas son contextualisme. Selon lui, les preuves empiriques montrent que la contribution de plusieurs expressions aux conditions de vérité des phrases dans lesquelles elles apparaissent varie selon le contexte.

4. Recanati concentre son attention sur l'utilisation assertive d'une phrase, mais sa théorie peut être étendue à d'autres usages. Je le suivrai en cela et me concentrerai sur les conditions de vérité des phrases assertives et tiendrai avec lui pour acquis qu'elles donnent sa signification à une phrase, lorsque celle-ci est utilisée.

5. Recanati précise son affirmation en soutenant que le contenu sémantique de presque toutes les utilisations de phrases est déterminé par le contexte, laissant la possibilité que, lors de leur utilisation, il y ait quelques cas où le contenu est déterminé par la signification lexicale des constituants de la phrase et par la forme logique de celle-ci, mais Recanati ne discute pas des cas de ce genre (p. 58). Il n'est pas évident qu'il prenne cette possibilité au sérieux. En tous les cas, il concentre son attention sur les déterminants contextuels de la signification.

6. Dans ce qui suit, je laisserai tomber " assertive » et parlerai seulement de l'utilisation des phrases, mais il devra être entendu que c'est des utilisations assertives dont il est question, à moins qu'il soit spécifié qu'il en est autrement. 
qu'il y a deux sens à " déterminer » et que, dans le sens qui est important, les processus pragmatiques invoqués par Recanati ne déterminent pas ce qui est dit dans les phrases utilisées. Je décrirai brièvement la théorie de Recanati et me pencherai ensuite sur quelques critiques de cette approche.

Recanati distingue trois sortes de signification : linguistique, primaire et secondaire (pp. 21 et 80). Les significations linguistiques ont trait aux types d'expressions, les significations primaires se rapportent à ce qui est dit par les locuteurs, et les significations secondaires à ce que les locuteurs sous-entendent, c'est-à-dire à l'implicature conversationnelle de ce qui est dit. Une théorie sur la signification, que Recanati appelle " minimalisme ${ }^{7}$, soutient que la signification d'une phrase déclarative est donnée par la signification linguistique des expressions qui la constituent et par sa forme logique, sauf si elle contient des indexicaux ou des variables. Les significations linguistiques des expressions d'une phrase et sa forme logique sont les caractéristiques des types de phrases et d'expressions. Donc, si l'occurence d'un type qui ne contient pas d'indexicaux est utilisée lors d'une occasion particulière, elle hérite des conditions de vérité de son type. Les indexicaux sont des éléments linguistiques qui peuvent changer de valeur sémantique d'un contexte à l'autre. Si un type de phrase utilisé dans un contexte particulier contient un indexical, ce qui selon la théorie minimaliste ne s'applique qu'à un petit nombre d'expressions, il est nécessaire que la valeur sémantique de l'indicateur indexical soit donnée par des caractéristiques du contexte dans lequel la phrase est utilisée. Une façon de voir la signification des indexicaux est de les considérer comme des fonctions qui vont du contexte d'utilisation à la valeur sémantique ${ }^{8}$. Quand je dis, par exemple :

1. "Je suis ici maintenant ",

les valeurs de «Je », de «maintenant » et de « ici » sont données par leur signification linguistique - respectivement, la personne qui utilise l'énoncéoccurrence, le moment de l'énoncé-occurence et l'endroit de l'énoncé-occurence -, de même que par le contexte dans lequel l'énoncé 1 est utilisé. Ces significations linguistiques sont respectivement attribuées, pour " Je ", à moi comme valeur ; pour " maintenant », au moment où l'énoncé 1 est utilisé, et pour " ici », à l'endroit où il est énoncé'. Pour produire la signification de 1 , en plus de la signification des expressions qu'elle contient, il est nécessaire de connaitre la forme logique de la phrase ; cette connaissance m'est donnée par la syntaxe de mon idiolecte $^{10}$. Ainsi, étant donné la signification des expressions et la forme logique de 1 , la sémantique de mon idiolecte génèrerait sa signification.

7. Voir Cappelen et Lepore (2005) pour une position minimaliste sur la signification.

8. Voir David Kaplan (1989) pour une théorie de ce genre.

9. Recanati ne traiterait pas les valeurs des indexicaux comme des objets en contexte d'utilisation, mais plutôt comme des significations, c'est-à-dire comme des représentations mentales dans l'esprit des locuteurs ou des allocutaires.

10. Pour compléter l'attribution de signification aux expressions de 1 , il faudrait donner des valeurs sémantiques à " être » et au présent de l'indicatif. 
Recanati rejette le minimalisme (pp. 7-8). Il pense plutôt que la signification linguistique et la forme logique ne sont jamais suffisants pour déterminer la signification d'une phrase utilisée dans un contexte, même si celle-ci ne contient pas d'éléments indexicaux. Pour Recanati, la proposition exprimée par l'utilisation d'une phrase - ce qui est dit en utilisant la phrase — résulte de deux processus interprétatifs contextuels : un processus ascendant [bottom up] obligatoire appelé "saturation ", déclenché par les éléments indexicaux dans la phrase, et des processus descendants [top down] optionnels, qui ne sont pas déclenchés de la même façon, comprenant l'enrichissement libre, la spécification, le renforcement, le transfert et la modulation. Le processus obligatoire est un processus interprétatif, lequel est nécessaire pour que l'énoncé ait des conditions de vérité ; le processus optionnel est un processus interprétatif qui n'a pas besoin d'être appliqué pour que l'énoncé ait des conditions de vérité, mais qui pourrait bien être nécessaire pour produire ce qui est signifié par l'énoncé dans le contexte de son utilisation.

Un exemple aidera à éclaircir certains de ces processus. Supposez qu'un vendeur, dans une boutique parisienne à la mode, utilise la phrase suivante :

2. Votre belle veste de sport rouge est prête.

Deux cas de saturation, nécessaires pour qu'il ait des conditions de vérité, s'appliquent à l'énoncé de la phrase 2. Premièrement, on doit ajouter à la phrase la spécification de ce pour quoi la veste est prête. Elle pourrait être prête à être achetée, à être emportée, à être montrée, à être réparée, etc. Une façon de contourner ce problème est de supposer que, dans la structure sousjacente à la phrase, il y a quelque chose comme " à $x$ ». Puisque cette phrase contient une variable, une valeur doit être fournie par le contexte dans lequel 2 est utilisée. Deuxièmement, puisque " votre » est un indexical, sa valeur doit aussi être donnée par le contexte dans lequel 2 est utilisé. Selon Recanati, il est obligatoire de fournir des valeurs pour "votre " et " $x$ » dans " à $x$ ", puisque là où il n'aurait pas de valeur, l'énoncé serait sans conditions de vérité. De plus, il y a d'autres processus reliés aux conditions de vérité de l'énoncé qui ne sont pas obligatoires et ne sont déclenchés ni par une variable ni par un indexical dans la phrase. Par exemple, une spécification supplémentaire peut être donnée par la relation, indiquée par le génitif de l'indexical "votre ", entre le référent de " votre » et celui de "la veste de sport rouge ». La personne à qui réfère " votre " pourrait être l'acheteur, le propriétaire, le designer, le couturier, etc., de la veste de sport, chacune de ces possibilités indiquant une relation différente entre les référents de "votre " et de "la veste de sport rouge ". De plus, le critère d'application de " rouge " dépend du contexte, puisque les objets peuvent être rouges de différentes façons, par exemple en étant rouge à l'extérieur, à l'intérieur ou en ayant des touches de rouge. Ainsi, la façon dont la veste est rouge peut encore être précisée, un processus appelé modulation, qui, selon Recanati, affecte les conditions de vérité de l'usage de 2. Ces processus ne sont pas indépendants. La valeur choisie pour la relation 
indiquée par le génitif est reliée à la valeur sélectionnée pour $x$ dans "à $x$ ", laquelle est à son tour reliée à la valeur donnée à "votre ". Par exemple, si une interprétation donne " un couturier ${ }^{11}$ » comme valeur de "votre ", alors une valeur plausible pour la variable "à $x$ » est " être réparée ».

Ces processus - saturation, spécification, modulation, etc. - produisent ce qui est dit par le locuteur lorsqu'il utilise la phrase. En contexte, toutefois, il est possible que le locuteur sous-entende plus que ce qu'il dit. Supposez que les vestes de sport rouges ne soient pas à la mode à Paris et considérées comme vulgaires dans les boutiques de vêtements chics, ce qui est connu de l'interprète, qui a aussi de bonnes raisons de croire que le vendeur le sait tout autant. L'interprète peut alors inférer que le vendeur est ironique en disant 2 , et qu'il ne considère pas que la veste de sport est belle. Il peut plutôt inférer que, en disant 2, le vendeur sous-entend ce qui est exprimé par :

3. Votre veste de sport rouge n'est pas belle ${ }^{12}$.

Ce n'est pas le seul sous-entendu qu'un interprète pourrait inférer de l'énoncé 2. Supposez que l'auditeur interprète 2 comme étant adressé à la personne qui achète la veste de sport et comme voulant dire que la veste est prête à être achetée. De cela, et d'autres faits à propos du contexte, il pourrait inférer que le vendeur sous-entend :

\section{L'acheteur devrait aller à la caisse et payer l'article.}

Les exemples 3 et 4 sont des cas d'implicature conversationnelle et incitent l'interprète à inférer ce que le locuteur de 2 sous-entend à partir de ce qu'il dit.

Pour Recanati, il y a une différence importante entre la façon dont une chose est dite (la signification linguistique primaire) et la façon dont ce qui est sous-entendu dans la conversation (la signification linguistique secondaire) sont déterminés. Il rejette la position selon laquelle les deux seraient déterminés de façon inférentielle (pp. 38-40). Recanati soutient que l'interprétation de ce qui est dit n'est pas le résultat d'une inférence consciente ou inconsciente, mais bien de processus locaux sub-personnels (p. 23). Il affirme que :

[...] l'interprétation qui émerge éventuellement et incorpore l'output de différents processus pragmatiques résulte d'un processus mécanique aveugle, lequel n'implique aucune réflexion de la part de l'interprète. C'est la dynamique de l'accessibilité qui fait tout, et aucune « inférence » n'est requise. En particulier, il n'y a nul besoin de considérer les croyances et les intentions du locuteur (p. 32).

L'accessibilité est donc la clé permettant de comprendre comment Recanati pense que la signification primaire de l'utilisation d'une phrase - ses conditions de vérité - est déterminée. Le processus commence avec l'activation,

11. Plus précisément, selon Recanati, la représentation d'un couturier.

12. La valeur sémantique de "votre " dans 3 doit être la même que dans 2 . 
dans l'esprit de l'interprète, des significations des expressions de la phrase interprétée, lesquelles sont attribuées aux types d'expression de l'idiolecte de l'interprète (p. 30). Ces significations, toutefois, ne sont pas les seules à être activées. Au même moment, d'autres significations le sont également : celles, nous dit Recanati, qui sont attribuées aux significations, encodées linguistiquement, des expressions de la phrase énoncée. Il est par conséquent possible que plus d'une signification soit attribuée à une expression. S'il y en a plus d'une, alors comment celle qui figure dans la signification de la phrase que l'auditeur interprète est-elle sélectionnée ? Selon Recanati, si un ensemble de significations sont activées, c'est la plus accessible, ou la plus saillante, qui joue un rôle dans l'interprétation. La saillance ou l'accessibilité sont affaire de degré. Elles peuvent être affectées par l'information contenue dans le contexte d'utilisation de l'expression, y compris par les significations d'autres expressions dans ce qui a été énoncé ou en vient à l'être (p. 30). De plus, l'information non linguistique, qu'elle soit contextuelle ou d'arrière-plan, peut jouer un rôle en faisant varier le degré d'accessibilité à la signification d'une expression. Pour Recanati, l'accessibilité ou la saillance de la signification d'une expression détermine mécaniquement la signification qu'une expression a dans un contexte particulier, une signification qui n'implique pas que l'interprète fasse une inférence faisant appel aux croyances ou aux intentions du locuteur ${ }^{13}$.

Deux sens de " détermine ", épistémologique et métaphysique, sont en jeu ici : un interprète, dans la compréhension de l'énoncé d'une phrase, détermine en un sens clairement épistémologique — c'est-à-dire, en vient à savoir — ce que l'énoncé signifie dans le contexte où il est utilisé. S'il n'en était pas venu à savoir ce qu'il signifiait, il ne l'aurait pas compris ${ }^{14}$. Il n'est pas clair, toutefois, qu'un interprète détermine métaphysiquement, — c'est-à-dire qu'il fixe - la signification d'un énoncé dans le contexte où il est utilisé, mais je crois que c'est ce que Recanati propose. Appelons cette dernière position « interprétationnisme ", soit la position selon laquelle la signification qu'un interprète attribue à l'utilisation d'une phrase fixe métaphysiquement la signification de celle-ci.

Considérer Recanati comme un interprétationniste pourrait sembler aller à l'encontre de sa position, selon laquelle " dire " est une sorte de signification non naturelle gricéenne (p. 13). Cela semble suggérer que la signification de ce qui est dit est fonction d'une intention gricéenne complexe du locuteur,

13. C'est une des questions sur lesquelles les théoriciens de la pertinence et Recanati divergent, puisque les théoriciens de la pertinence soutiennent que la signification primaire, ou " explicature ", comme ils l'appellent, est le résultat d'une inférence de la part de l'interprète (cf. Carston, 2002). Cf. Recanati (38-48) pour une discussion sur la différence entre ses positions et la théorie de la pertinence sur la question de savoir comment les auditeurs parviennent à la signification primaire.

14. Je ne pense pas que la compréhension au sens strict soit nécessaire à la communication. Dans la plupart des situations de communication, les auditeurs n'ont pas besoin de comprendre ce que l'occurrence qu'ils traitent signifie. Tout ce qui est nécessaire pour eux, c'est d'être assez près de comprendre ce que quelqu'un dit pour poursuivre la conversation. 
y compris de l'intention que son auditoire reconnaisse son intention ${ }^{15}$. Ainsi, il semblerait que Recanati soutienne que ce n'est pas l'interprétation de l'allocutaire qui fixe la signification d'un énoncé dans un contexte, mais l'intention gricéenne du locuteur ${ }^{16}$. Il y a là une certaine vérité, mais cela ne nous empêche pas de considérer Recanati comme un interprétationniste. Il prétend que concevoir l'énonciation comme une espèce de signification non naturelle gricéenne implique que :

[c]e qui est dit (comme ce qui est signifié en général, y compris ce qui est sousentendu) doit être exposé au regard public. Il en est ainsi parce que la signification non naturelle est essentiellement une affaire de reconnaissance d'intention. Selon cette théorie, ce qui est dit par l'utilisation d'une phrase dépend des intentions publiquement reconnaissables du locuteur et peut difficilement en être séparé. De là mon "principe de disponibilité ", selon lequel «ce qui est dit " doit être pleinement analysé en accord avec les intuitions partagées par ceux qui comprennent pleinement l'énoncé — particulièrement le locuteur et l'auditeur, dans un cadre normal (italique de l'auteur, p. 14) ${ }^{17}$.

\section{Ce qui est dit implique donc le principe de disponibilité de Recanati.}

Ce qui est dit doit être intuitivement accessible aux participants à la conversation (à moins que quelque chose n'aille pas et qu'ils ne comptent pas comme des «interprètes normaux ») (p. 20).

C'est-à-dire que ce qui est dit doit être accessible à la fois au locuteur et à l'allocutaire. Ce qui détermine ce qui est dit — c'est-à-dire qui le fixe métaphysiquement - est donc accessible à la fois au locuteur et à l'allocutaire. Donc, si l'on considère sa position sur ce qui fixe ce qui est dit du point de vue de l'allocutaire, alors Recanati est un interprétationniste ${ }^{18}$.

15. Il est difficile de concilier l'affirmation de Recanati selon laquelle dire est une espèce de signification non naturelle gricéenne, qui implique une intention complexe gricéenne que l'auditeur reconnaisse cette intention, avec l'affirmation de Recanati selon laquelle pour que l'interprète comprenne le locuteur, "il n'y a nul besoin de considérer les croyances et les intentions du locuteur " (p. 32) et donc nul besoin pour l'interprète de reconnaître l'intention gricéenne du locuteur pour comprendre ce dernier. Ainsi, selon Recanati, il serait parfaitement possible que les intentions gricéennes des locuteurs ne soient jamais reconnues et que néanmoins les interprètes comprennent ce qu'ils disent. Si c'est bien le cas, il est difficile de voir en quoi il est pertinent de supposer que les locuteurs ont de telles intentions complexes gricéennes, puisqu'elles ne semblent jouer aucun rôle dans la communication de ce que Recanati appelle la signification primaire.

16. Ce point a été souligné par Michel Seymour (communication personnelle).

17. Il est important de remarquer comment Recanati utilise le mot " analysé ", dans ce passage. Si ce qui est dit peut être « analysé » du point de vue de l'interprétation par l'auditeur, alors la compréhension par l'auditeur de ce qui est dit détermine dans un sens métaphysique ce qui est dit.

18. Il peut sembler étrange de soutenir que ce peut être l'interprète qui fixe ce qui est dit, c'est-à-dire que son interprétation ne détermine pas seulement de façon épistémologique, mais aussi métaphysique, ce qui est dit. On pense que, s'il existe quelque chose qui fixe métaphysiquement ce qui est dit, alors ce sont les faits concernant le locuteur, puisque que c'est dans les faits qu'il dit ce qu'il dit, que ce qui est dit est dit. Mais si on donne aux faits concernant le locuteur la priorité quant à ce qui est dit métaphysiquement, alors les processus pragmatiques que Recanati attribue à l'interprète font partie, au mieux, d'une théorie de la compréhension et n'ont 
Il est problématique d'interpréter ce qui est dit — la signification d'un énoncé dans le contexte de son utilisation - en faisant appel à ce qu'un interprète comprend qu'il signifie. Les interprètes, comme le reconnaît Recanati, peuvent faire des erreurs. Prenons l'exemple suivant ${ }^{19}$. Supposez que deux chiens gambadent sur la pelouse : un grand saint-bernard à l'avant-plan, et un petit épagneul cocker à l'arrière-plan, partiellement caché par le plus grand chien. J'observe les deux chiens, en compagnie de mon ami Sam, qui ne sait pas que j'ai un chien. Les deux chiens sont visibles par nous deux et, le plus important, ils sont dans mon champ de vision - ce qui est évident pour Sam et nous sommes tous les deux francophones de naissance. Sans pointer du doigt, je dis :

\section{C'est mon chien.}

En utilisant «c'est » dans 5, je veux faire allusion à l'épagneul cocker, mon chien, et je veux utiliser les mots que je prononce dans leur sens ordinaire. Il est évident que ce que je dis est vrai seulement dans le cas où l'épagneul cocker qui gambade sur la pelouse est mon chien. Comment Sam, à qui s'adresse la remarque, interpréterait-il la signification de 5 ? Supposons que le prédicat de 5 ne pose aucun problème pour l'interprétation de mon énoncé, et concentrons notre attention sur « ce », auquel Sam doit attribuer une valeur sémantique pour l'interpréter. Sam connaît la signification de l'expression que j'énonce et accède à la signification de «ce ». Supposons que dans le lexique de l'idiolecte de Sam, une de ses significations soit l'objet perceptuellement saillant pour le locuteur au moment de l'énoncé de "c'est». Le prédicat de la phrase dirigerait l'attention de Sam vers les chiens qui sont dans mon champ visuel, qu'il suppose être similaire au sien, étant donné la façon dont lui et moi sommes situés par rapport à la scène. Puisque le saint-bernard est à l'avantplan de notre champ visuel commun et qu'il est plus proéminent que l'épagneul cocker, la valeur sémantique qui est activée ${ }^{20}$ pour "ce " dans l'interprétation que ferait Sam de mon énoncé en 5 serait une représentation

rien à voir avec la détermination métaphysique de ce qui est dit, comme Recanati semble l'affirmer tout au long de son livre. Voir, par exemple, le diagramme de la page 21, qui présente la théorie de la disponibilité de Recanati et dans lequel il semble y avoir un lien explicite entre les processus pragmatiques qu'il attribue au locuteur et la fixation métaphysique de ce qui est dit.

19. Recanati soutient que, pour susciter des intuitions sur les conditions de vérité, " on doit simplement fournir aux sujets des scénarios décrivant des situations, ou mieux, des images — possiblement animées — de situations, et leur demander d'évaluer l'énoncé cible comme vrai ou faux au regard de la situation en question » (p. 15). La façon dont cette méthode produit les conditions de vérité de ce qui est dit n'est pas claire, puisqu'elle ne nous dit pas ce qui, dans la situation, détermine la vérité de l'occurrence cible. Tout ce qu'elle nous dit, c'est si le locuteur ou l'auditeur considère l'énoncé comme vrai, sans nous dire ce que sont les conditions de vérité. Je suppose que le scénario que je m'apprête à décrire est conforme aux conditions posées par Recanati pour les scénarios censés susciter les intuitions des locuteurs et des auditeurs quant aux conditions de vérité.

20. J'adopte ici le langage de Recanati pour décrire comment l'interprétation fonctionne, mais je ne m'engage pas envers son explication " mécanique " de l'interprétation. 
du saint-bernard plutôt que de l'épagneul cocker ${ }^{21}$. C'est-à-dire que le saintbernard est plus saillant ${ }^{22}$ que l'épagneul cocker dans notre espace perceptuel commun et donc qu'il lui est plus accessible que ce dernier pour attribuer une valeur sémantique à " ce ». Il n'y a dans cette situation aucune autre information accessible qui puisse être utilisée " mécaniquement » ou autrement pour que Sam détermine - c'est-à-dire, en vienne à connaître — la valeur sémantique de " c'est » dans mon énoncé. Je n'ai pas pointé du doigt vers l'épagneul cocker, et l'orientation de ma tête est telle qu'on peut tenir pour acquis que les deux chiens se trouvent dans mon champ visuel. De plus, Sam ne sait même pas, par hypothèse, que j'ai un chien et donc que j'ai un épagneul cocker. Selon la théorie de l'accessibilité de Recanati, la condition de vérité que Sam attribuerait donc à mon énoncé serait que celui-ci n'est vrai que si le saintbernard est mon chien. Ce faisant, il ferait une erreur et n'aurait pas compris ce que j'ai dit, puisque ce que j'ai dit n'est vrai que si l'épagneul cocker est mon chien.

Recanati, qui considère un exemple similaire, s'en tire en abandonnant l'interprète actuel comme déterminant des conditions de vérité et en lui substituant ce qu'il appelle l' « interprète normal » (p. 19). Selon Recanati, un interprète normal en est un qui

[...] sait quelle phrase a été énoncée, connaît la signification de cette phrase, connaît les faits contextuels pertinents (vers qui l'on pointe, et ainsi de suite). Les utilisateurs ordinaires du langage sont des interprètes normaux, dans la plupart des situations [...]. Mais il $\mathrm{y}$ a des situations [...] où les utilisateurs actuels font des erreurs et ne sont pas des interprètes normaux. Dans de telles situations, leur interprétation ne fixe pas ce qui est dit. Pour déterminer ce qui est dit, nous devons regarder l'interprétation qu'un interprète normal donnerait (italique de l'auteur, p. 19-20).

Puisque Sam a fait une erreur, il n'est pas un interprète normal, au sens étendu que Recanati donne à cette notion, et l'interprétation qu'il donne doit être rejetée et remplacée par ce qu'un interprète normal interpréterait comme

21. Puisque la théorie de la signification de Recanati est une théorie représentationnelle, je considère que la valeur sémantique de «ce » dans ce scénario n'est pas le saint-bernard luimême, mais une représentation du saint-bernard.

22. Nous pouvons considérer la saillance d'un objet visuel en fonction de la place et de l'espace qu'il occupe dans le champ visuel, entre autres propriétés. Les objets à l'avant-plan sont plus saillants que les objets à l'arrière-plan, et les objets qui couvrent une plus grande partie du champ visuel sont plus saillants que ceux qui couvrent une moins grande partie du même champ visuel. Dans les deux critères de saillance, le saint-bernard est plus saillant que l'épagneul cocker. Le saint-bernard est à l'avant plan du champ visuel, l'épagneul cocker est à l'arrière-plan, et le saint-bernard occupe une plus grande partie du champ visuel que l'épagneul cocker. L'avant-plan ou l'arrière-plan, et l'espace occupé dans le champ visuel ne sont pas les seules propriétés qui peuvent jouer un rôle dans la saillance. La couleur, le mouvement et les propriétés liées à d'autres modalités sensorielles, par exemple, peuvent jouer un rôle pour déterminer quel objet est saillant. Dans l'exemple considéré ici, l'avant-plan ou l'arrière-plan, et l'étendue du champ visuel occupé déterminent la saillance. 
étant la signification de mon énoncé. Pour Recanati, un interprète normal est un interprète qui ne fait pas d'erreurs ${ }^{23}$. C'est-à-dire un interprète qui, dans toutes les situations, donne l'interprétation correcte à ce qu'un locuteur dit, et l'interprétation qu'il donne constitue la signification de ce qui est dit.

Plusieurs problèmes se posent quant à l'interprète normal de Recanati, le premier étant un problème interne à sa théorie. Selon lui, les interprétations qui produisent les significations primaires - ce que je dis en énonçant 5 ne sont pas inférentielles et ne font pas non plus appel aux intentions ou aux croyances du locuteur. Pour interpréter 5, toutefois, on croirait que son interprète normal doit faire appel à ce à quoi j'ai l'intention de référer en utilisant «ce " dans 5, pour fixer une valeur sémantique au démonstratif puisque, dans la situation que je décris, la caractéristique de la situation la plus plausible pour fixer la valeur sémantique de mon usage de «ce » est mon intention. Si cela n'est pas mon intention, il en revient à Recanati de fournir une explication de ce qui fixe la représentation du référent du démonstratif.

Le deuxième problème est que Recanati n'explique pas comment son interprète normal procéderait pour comprendre ce que je dis. Il nous dit qu'un tel interprète saurait un bon nombre de choses pertinentes à la compréhension de ce que je dis. Il ne suffit pas, dans une théorie de l'interprétation, de dire qu'un interprète sait ce qu'il est nécessaire de savoir pour interpréter un énoncé. Ce que cette théorie doit nous dire, c'est comment cette interprétation est effectuée, c'est-à-dire comment l'interprète en vient à savoir ce qui est pertinent pour son interprétation. Sam utilise de façon correcte l'information qui lui est accessible dans le contexte et, compte tenu de cette information, donne de ce que je dis l'interprétation la plus plausible, bien qu'erronée. La question est de savoir comment l'interprète normal de Recanati, s'il était dans la même situation que Sam, en viendrait à donner l'interprétation correcte de ce que je dis, ce que Recanati n'explique pas. Quelle information, non disponible pour Sam, pourrait-il utiliser pour atteindre l'interprétation correcte ? La télépathie est bien sûr exclue. Recanati pourrait peut-être dire que son interprète normal n'aurait qu'à me demander ce à quoi je fais référence pour arriver à l'interprétation correcte. Mais ce n'est pas acceptable, pour deux raisons. Recanati exclut du champ d'information qu'un interprète peut utiliser un énoncé qui fait appel aux intentions du locuteur. De plus, si l'information quant à mes intentions était ajoutée à l'information à laquelle un interprète normal peut faire appel, cela laisserait inexpliquée la façon dont ce que je dis pourrait avoir une valeur sémantique dans les situations où cette information n'est pas disponible, situations tout à fait normales.

La troisième difficulté dans la théorie de Recanati est que dans certaines utilisations de phrases il est difficile de voir comment cette théorie

23. Il est étrange d'appeler « normal » un tel interprète, puisque les interprètes normaux ordinaires font des erreurs. Dans la situation que j'ai décrite, Sam est un interprète parfaitement normal, bien qu'il ait fait une erreur. 
s'applique. Tout au long de Litteral Meaning, Recanati adhère au contextualisme, qu'il considère être le point de vue selon lequel « [...] les phrases ellesmêmes expriment un contenu déterminé seulement dans le contexte d'un acte de langage » (p. 159). Dans le dernier chapitre, il élargit la portée de sa théorie pour y inclure les " actes de pensée ». Il nous dit que :

[...] les actes de langage (ou actes de pensée) dans le contexte desquels on dit que les phrases véhiculent des contenus déterminés n'ont pas besoin d'être compris de façon trop restrictive. La liste comprend : supposer que $p$, faire comme si on disait $p$ et affirmer que $p$ (p. 155).

Étendre sa théorie de l'interprète normal pour qu'elle comprenne de tels actes force Recanati à adopter la position selon laquelle ce qui est supposé par un locuteur doit être assimilé à ce qu'un interprète normal comprendrait comme étant supposé, dans le contexte en question, et de même pour ce qui est de faire comme si on disait (p. 19). Recanati ne nous dit pas jusqu'où on peut étendre le champ des actes de pensée, mais je suppose que celui-ci doit inclure toute pensée dans laquelle apparaît une phrase dont le contenu est défini. Si je pense la phrase :

6. Je suis fatigué.

et que, ce faisant, je pense que je suis fatigué, alors 6 a un contenu sémantique dans le contexte de mon acte de pensée ${ }^{24}$. Ce que je pense, qui est exprimé par 6 dans le contexte de mes pensées, n'est vrai que dans le cas ou je suis fatigué. Cela pose un problème pour l'explication que Recanati donne de ce qui fixe le contenu sémantique d'une phrase en contexte. Son interprétationnisme ne semble pas s'appliquer à de telles phrases. Je suppose que l'interprète normal de Recanati n'a pas accès à ce que les autres pensent, à moins qu'ils disent ou fassent quelque chose qui indique ce qu'ils pensent. Nous avons exclu la télépathie comme source d'interprétation.

Une façon de contourner le problème est peut-être d'amender la théorie de l'interprète normal de Recanati de façon à ce qu'elle puisse s'appliquer à des phrases pensées. Nous pouvons dire qu'une phrase pensée a le contenu sémantique qu'un interprète normal comprendrait si elle avait été dite plutôt que pensée. Ainsi, un interprète normal comprendrait 6 comme ayant le contenu que je suis fatigué, puisque si j'avais énoncé cette phrase, c'est ce qu'un interprète normal aurait compris qu'elle signifie. La difficulté, dans cette façon de s'en sortir, c'est qu'il y a des phrases qui ne posent pas problème lorsqu'elles sont pensées, mais qui en tant qu'actes de langages semblent s'autoréfuter, par exemple :

7. Je n'émets aucun son.

Il n'y a aucun problème à penser cette phrase et, en la pensant, je n'émets aucun son. De plus, si je ne dis rien, ce que je pense est vrai. Mais l'énoncer est une

24. Il ne s'agit pas ici de suggérer que chaque fois que j'ai une pensée, même une pensée occurrente, il y a une phrase qui me passe par la tête et dont le contenu est le contenu de ma pensée, mais plutôt de suggérer de tels cas existent. 
toute autre affaire. Si je l'énonçais, alors un interprète normal, et même l'interprète normal de Recanati, ne considérerait pas que je parle littéralement, puisque mon énoncé se réfute lui-même. Les conditions de vérité qu'un interprète attribuerait à l'utilisation de la phrase pour la comprendre ne seraient donc pas les conditions de vérité qu'a la phrase dans le contexte de la pensée. Conséquemment, il s'agit d'une phrase utilisée dans le contexte de pensée pour lequel la théorie de Recanati ne peut fournir de contenu sémantique. Peut-être Recanati pourrait-il dire que sa théorie n'est pas faite pour prendre en considération les phrases pensées, mais seulement les phrases produites dans le cadre d'actes de langage. Si c'était sa réponse, alors sa théorie serait incomplète comme explication de ce qui fixe le contenu sémantique des phrases lorsqu'elles se produisent dans différents types de contextes. La pensée selon laquelle je n'émets aucun son a un contenu sémantique dont une théorie du contenu sémantique doit rendre compte.

Le quatrième problème, et sans doute le plus sérieux, c'est que Recanati invoque un interprète normal pour sauver sa théorie, et cela fait de son interprétationnisme une théorie non empirique, puisqu'elle rend son explication imperméable aux preuves contraires. Recanati expose une théorie de l'interprétation qui est censée fournir les valeurs sémantiques des utilisations de phrases en contexte. Si la théorie mène à ce que l'on croit être une attribution incorrecte des conditions de vérités pour l'utilisation d'une phrase, Recanati a beau jeu de dire que, dans un tel cas, l'interprète invoqué n'est pas un interprète normal, puisque, par définition, un interprète normal est quelqu'un qui ne fait pas d'erreurs d'interprétation. Sa théorie n'est donc pas une théorie empirique ${ }^{25}$.

Il résulte vraisemblablement des considérations précédentes que l'interprétationnisme doit être abandonné. C’est-à-dire que l'interprétation qu'un interprète normal fait de l'utilisation d'une phrase ne fixe pas la signification de cette utilisation de la phrase. Cela veut dire que les processus pragmatiques, i.e. les processus psychologiques dont Recanati affirme qu'ils se produisent dans l'interprétation, ne jouent aucun rôle dans la fixation de la signification primaire de l'utilisation des phrases. De plus, le principe de disponibilité de

25. Recanati défend sa théorie de l'accessibilité contre une critique similaire à la mienne, formulée par Dan Sperber, selon laquelle : «parfois, la première interprétation qui nous vient à l'esprit (la plus accessible) s'avère insatisfaisante et force l'auditeur à revenir en arrière » (p. 32). Pour contrer cette objection, Recanati suppose que l'auditeur ne donne pas qu'une seule interprétation, mais une série d'interprétations, lorsqu'il réalise que son interprétation initiale est erronée. Supposons que l'interprétation initiale pour une utilisation de $S$ est $s$, qui est erronée, mais qu'à un stade subséquent de la conversation, ça ne soit pas $s$ qui est la plus accessible, mais s'. À ce stade subséquent, il y a donc eu un changement d'accessibilité, s' étant devenu plus accessible que $s$. C'est alors s' qui est censée fournir le contenu sémantique de l'utilisation de $S$. La réponse de Recanati à Sperber n'a pas de prise sur mon contre-exemple à la théorie de Recanati, basé sur 5. Des interprétations successives autres que celle qu'il donne ne peuvent être données par Sam puisqu'aucune information ne lui est accessible et ne peut le mener à l'interprétation correcte. 


\section{4 · Philosophiques / Printemps 2006}

Recanati doit aussi être abandonné puisqu'il s'appuie sur la notion d'interprète normal, pour laquelle on ne nous fournit pas d'explication.

Qu'en est-il alors de la théorie de Recanati ? Pourrions-nous abandonner son interprétationnisme et nous tourner vers le locuteur et ses intentions complexes comme déterminant de la signification primaire ? Le problème, quant à cette tentative de sauver sa théorie, c'est qu'à l'exception des intentions complexes gricéennes attribuées aux locuteurs, Recanati nous dit peu de choses sur les processus psychologiques en jeu dans la production du discours. Les processus pragmatiques qui sont centraux dans la théorie de Recanati sont les processus psychologiques censés rendre compte de l'interprétation, mais ils ne peuvent être attribués d'aucune façon évidente à la production du discours. De plus, et c'est encore plus important, je ne crois pas que les intentions gricéennes du locuteur peuvent faire ce travail. Rappelezvous que Recanati considère l'énonciation comme une espèce de signification gricéenne non naturelle. Selon ce point de vue, pour qu'un locuteur dise quelque chose, il doit avoir une intention complexe gricénne, laquelle inclut l'intention que son auditoire la reconnaisse. Le problème est qu'un locuteur-penseur peut utiliser une phrase sans avoir d'intention gricéenne envers un auditoire. Il peut utiliser la phrase en se parlant à lui-même ou avoir une pensée qui entraîne l'occurrence mentale d'une phrase. Dans ni l'un ni l'autre de ces cas il n'a l'intention que son auditoire reconnaisse son intention de dire quelque chose, puisqu'il ne considère pas avoir d'auditoire. La conclusion qu'on peut en tirer est que la théorie de Recanati, qu'elle soit considérée du point de vue de l'auditeur ou de celui du locuteur, ne fournit pas d'explication de ce qui fixe la signification de l'utilisation des phrases ${ }^{26}$.

\section{Références}

Carston, Robyn. Thoughts and Utterance : the Pragmatics of Explicit Communication, Oxford, Blackwell, 2002.

Grice, H. P. Studies in the Way of Words, Cambridge, Mass., Harvard University Press, 1989.

Kaplan, David. "Demonstratives ", in J. Almog, H. Wettstein et J. Perry (dir.), Themes from Kaplan, New York, Oxford University Press, 1989.

Cappelen, Herman et Ernest Lepore (2005). Insensitive Semantics : in Defense of Semantic Minimalism and Speech Act Pluralism,Oxford, Blackwell, 2005.

Recanati, François. Literal Meaning, Cambridge, Cambridge University Press, 2004. Sperber, Dan et Wilson Deirdre. Relevance : Communication and Cognition, Oxford, Blackwell, 1986.

26. J'aimerais remercier Eros Corazza, Michel Seymour, Jeffery Speaks et Robert Stainton pour leurs commentaires utiles sur cet article. 